British Journal of Nutrition (2022), 128, 352-360

doi:10.1017/S0007114521003238

(C) The Author(s), 2021. Published by Cambridge University Press on behalf of The Nutrition Society. This is an Open Access article, distributed under the terms of the Creative Commons Attribution licence (http://creativecommons.org/licenses/by/4.0/), which permits unrestricted re-use, distribution, and reproduction in any medium, provided the original work is properly cited.

\title{
The effect of aspartame and sucralose intake on body weight measures and blood metabolites: role of their form (solid and/or liquid) of ingestion
}

\author{
Marie-Elizabeth E. Ragi, Rachelle El-Haber, Fidele El-Masri and Omar A. Obeid* \\ Department of Nutrition and Food Sciences, Faculty of Agricultural and Food Sciences, American University of Beirut, \\ Beirut 1107 2020, Lebanon
}

(Submitted 16 May 2021 - Final revision received 17 August 2021 - Accepted 18 August 2021 - First published online 23 August 2021)

\section{Abstract}

The ingestion of non-caloric sweeteners (NCS) from food and/or drink was intended to reduce caloric intake without compromising palatability. However, the inconclusive relation between NCS and body weight may partially relate to their form of ingestion (solid or liquid). Thus, two paralleled experiments (aspartame and sucralose) were conducted. In each, Sprague Dawley rats (7-week-old male) were randomly divided into four groups. In Expt 1, aspartame (0.05\%) was added to the diet (AD) or drinking water (AW) or both diet and water (ADW), and a control group (C) was given a non-sweetened diet with plain water. In Expt 2, sucralose (0.016\%) was similarly provided in the diet (SD) or drinking water (SW) or both diet and water (SDW), with a control group (C). All rats had free access to food and water for 7 weeks. Energy intake, body weight and body composition were monitored and blood metabolites were determined. Results showed that aspartame ingestion significantly increased body weight and fat mass mainly due to an increase in energy efficiency. The effect was related to the amount rather than the form of ingestion. Additionally, aspartame ingestion was associated with glucose intolerance. Sucralose ingestion had a similar impact to that of aspartame though to a lesser extent. In conclusion, 7-week ingestion of aspartame and sucralose had adverse effects on body measures that were not related to the form of ingestion.

Key words: Non-caloric sweeteners: Aspartame: Sucralose: Lean body mass: Body fat

In the past decades, the increased consumption of sugarsweetened beverages has been paralleled by an increase in weight gain, thus implicated in the development of obesity and its related diseases ${ }^{(1-5)}$. As a result, efforts directed towards the prevention of obesity, type 2 diabetes and CVD have been focused on lowering the consumption of sugar-sweetened beverages $^{(3,4)}$. Surprisingly, the decline in the availability and intake of total added sugar was not associated with a decrease in the prevalence of obesity, which sustained its rise in the last couple of decades ${ }^{(6,7)}$.

The use of non-caloric sweeteners (NCS) has been established as a replacement strategy for sugar in sweetened beverages, notably in soft drinks. With an intense sweetening power and very low caloric contribution, they bring the sweet taste without the extra empty energy content and harmful effects attributed to sugars. In short-term studies, swapping sucrosecontaining beverages with non-caloric beverages was shown to reduce weight gain and fat accumulation without resort to energy restriction ${ }^{(8,9)}$. However, some long-term studies showed a positive dose-response association between artificially sweetened beverages consumption with weight gain, incidence of type 2 diabetes and incidence of $\mathrm{CVD}^{(10,11)}$. These findings were proposed to relate to disturbances in the association between sweetness and energy intake. Normally, the sweet taste is thought to predict the caloric content of food. However, when sweetness is not accompanied by caloric intake, an adaptive mechanism is activated to compensate for caloric deficiency by increasing energy intake and/or diminishing energy expenditure ${ }^{(12)}$.

The relationship between sweetened (both caloric and noncaloric) beverages and body composition measures is far from being conclusive. The contrasting evidence mentioned above may suggest the involvement of other factors, such as ingestive behaviour. The form in which sweeteners are ingested, whether from solid food or liquid beverages, influences the metabolic processes. Indeed, energy-containing beverages were reported to elicit little dietary compensation as compared with solid food and may consequently lead to an increase in energy intake and a subsequent weight gain ${ }^{(13)}$. Therefore, we investigated, over a 7-week experimental period, the effect of NCS (aspartame and sucralose) ingestion from the diet (solid) and/or drinking beverages (liquid) on energy balance, body composition, and measures of metabolism in male Sprague Dawley rats.

Abbreviations: NCS, non-caloric sweeteners.

* Corresponding author: Omar A. Obeid, email omar.obeid@aub.edu.lb 


\section{Methods}

\section{Animal model}

The experimental protocol (no. 18-02-453) was approved by the Institutional Animal Care and Use Committee (IACUC) of the American University of Beirut, Lebanon. The study was performed following the criteria outlined in the Guide for the Care and Use of Laboratory Animals. Male Sprague Dawley rats (7 weeks old, Animal Care Facility, American University of Beirut) were housed individually in wire-bottom cages in a temperature $\left(22 \pm 1^{\circ} \mathrm{C}\right)$ and light (reverse light cycle $12 \mathrm{~h}$ dark-12 h light, lights off at 10:00 h) controlled room. Food and liquids were offered ad libitum in containers and bottles easily accessible for the rats.

\section{Experimental design}

Rats ( $n$ 48) were placed on a 1-week adaptation period, with free access to plain water and semi-synthetic AIN-93G-based diet (online Supplementary Table 1) to familiarise them with the environment and diet.

They were subsequently randomly divided into seven experimental groups, analysed as two separate experiments with the same control group. The amounts of sweeteners (0.05\% aspartame and $0.016 \%$ sucralose) used were set to equal the sweetness level of $10 \%$ sucrose solution, a common level of sweetness in beverages. The corresponding percentages $0.05 \%$ of aspartame and $0.016 \%$ of sucralose - were added weight per weight in both diet and drink.

Expt 1. Effect of aspartame ingestion from water and/or diet on body weight measures and blood metabolites:

- Group C control ( $n$ 6): Rats were maintained on unsweetened starch-based diet and plain water

- Group $\mathrm{AD}(n 7)$ : Rats were maintained on aspartame-sweetened diet and plain water

- Group AW ( $n$ 7): Rats were maintained on unsweetened starch-based diet, with aspartame-sweetened water offered for $12 \mathrm{~h}$, followed by $12 \mathrm{~h}$ of plain water

- Group ADW ( $n 7)$ : Rats were maintained on aspartame-sweetened diet, and aspartame-sweetened water offered for $12 \mathrm{~h}$, followed by $12 \mathrm{~h}$ of plain water

Expt 2. Effect of sucralose ingestion from water and/or diet on body weight measures and blood metabolites:

- Group C control ( $n$ 6): Rats were maintained on unsweetened starch-based diet and plain water.

- Group SD ( $n$ 6): Rats were maintained on sucralose-sweetened diet with plain water

- Group SW ( $n$ 7): Rats were maintained on unsweetened starch-based diet, with sucralose-sweetened water offered for $12 \mathrm{~h}$, followed by $12 \mathrm{~h}$ of plain water

- Group SDW ( $n$ 7): Rats were maintained on sucralose-sweetened diet, and sucralose-sweetened water offered for $12 \mathrm{~h}$, followed by $12 \mathrm{~h}$ of plain water
Rats were fed ad libitum their respective diets and beverages (online Supplementary Table 1) for 7 weeks. In the groups where the rats were provided with a sweetened beverage (AW, ADW, SW and SDW), the respective sweetened water was only available for $12 \mathrm{~h}$ per d (dark phase), after which they were switched to regular drinking water to avoid potential osmolarity disturbances.

On the day of killing, overnight fasted rats were anesthetised with inhaled isoflurane (Forane®, Abbott,) administered at $4 \%$ concentration $(0.8 \mathrm{ml}$ in a 41 container). Blood was collected from the superior vena cava and rats were euthanised by severing their hearts. The livers, epididymal adipose tissue, kidneys and hearts were immediately excised, weighed, frozen in liquid $\mathrm{N}_{2}$ and stored at $-80^{\circ} \mathrm{C}$ pending analysis. Blood samples were centrifuged at $2200 \mathrm{~g}\left(3^{\circ} \mathrm{C}\right)$ for $15 \mathrm{~min}$, and aliquots of plasma were collected and stored at $-80^{\circ} \mathrm{C}$ awaiting analysis.

\section{Food and fluid intakes, body weight and composition}

Food and fluid intakes (difference in the weight of the food containers and water bottles) were measured twice per week. Body weight and body composition were assessed weekly using NMR minispec (LF110 BCA analyzer, Bruker). Body weight, lean body mass and body fat mass were expressed as gross weight. In order to minimise the impact of the variations in initial body weight within the groups, weight gain was presented by determining the changes from baseline. Energy stored was calculated as the energy retained in the body per $100 \mathrm{~kJ}$ of energy consumed $(\mathrm{kJ} / 100 \mathrm{~kJ})$, while energy expenditure was estimated from the total energy intake and changes in body mass and composition $^{(14)}$.

Livers were freeze-dried (FreeZone 6 Freeze Dryer, LABCONCO) for $48 \mathrm{~h}$, and hepatic fat content was determined by lipid extraction with petroleum ether $\left(40-60^{\circ} \mathrm{C}\right)$ as solvent using an ANKOM XT10 extractor (ANKOM Technology). All determinations were carried out in duplicate.

\section{Plasma analysis}

Fasting plasma glucose, total cholesterol, HDL-cholesterol, TAG, albumin, plasma urea nitrogen and creatinine were determined with Vitros 350 Chemistry System (Ortho-Clinical Diagnostics, Raritan). Plasma insulin concentration was measured by enzyme immunoassay using the Rat/Mouse Insulin ELISA Kit (EZRMI-13K) (EMD Millipore Corporation). The Homeostatic Model Assessment for Insulin Resistance (HOMA-IR) was calculated from the values of fasting plasma glucose and insulin $^{(15)}$, by the formula: HOMA-IR = fasting plasma glucose $(\mathrm{mmol} / \mathrm{l}) \times$ fasting serum insulin $(\mathrm{mU} / \mathrm{l}) / 22 \cdot 5$.

\section{Statistical analysis}

The required number of rats was calculated using previously determined weight gain data $(6.0 \pm 0.95 \mathrm{~g} / \mathrm{d})$ and assuming a $25 \%$ difference in the mean, with a statistical power of $90 \%$ and a $5 \%$ significance level. Data were expressed as the mean and standard deviation of all values. SPSS Statistics 25.0 software (IBM Corp.) was used for statistical analysis. For both experiments, one-way ANOVA with sweetener type as a factor was 
performed. Multiple-way ANOVA (general linear model), using sweetener type and time as fixed factors, was used to analyse the results throughout the 7-week experimental period. In addition, Pearson's correlation was performed to determine the relationship between the amounts of NCS ingested (aspartame or sucralose) with the different parameters assessed.

\section{Results}

\section{Expt 1: Effect of aspartame ingestion from water and/or} diet on body weight measures and blood metabolites

Body weight gain increased with time $(P=0.001)$ and showed a significant difference between groups $(P=0.001)$; it was found significantly higher in the ADW group as compared with the control group (Fig. 1(a)). A similar pattern was observed for fat mass gain, in which the significance between groups $(P=0 \cdot 001)$ was highly attributed to a difference between the ADW and control groups (Fig. 1(b)). Lean body mass gain was significantly different according to time $(P=0.001)$ and groups $(P=0.019)$, with that of group ADW found lower than the control group but higher than that of group AW (Fig. 1(c)). In order to account for the amount of aspartame ingested per rat (Table 1) on body composition, a correlation was performed between ingested aspartame (whether from water and/or diet) and body weight measures. Interestingly, the amount of ingested aspartame was positively correlated with weight gain $(r=0.492$, $P=0.009)$, fat mass gain $(r=0.430, P=0.025)$ and energy efficiency $(r=0 \cdot 395, P=0 \cdot 041)$

At the energy balance level, energy intake $(P=0.496)$, expenditure $(P=0 \cdot 601)$ and efficiency $(P=0 \cdot 107)$ were similar between the groups (Table 1). However, energy efficiency of the ADW was slightly higher and a significant positive correlation was found with the amount of ingested aspartame $(r=0.410$, $P=0.034)$. Total fluid intake $(P=0.409)$ was not affected by aspartame ingestion. The amount of aspartame consumed was, however, significantly different, highest when the sweetener was added to both diet and drink (group ADW), followed by group $\mathrm{AD}$ with aspartame in the diet, then lowest in group $\mathrm{AW}$ when aspartame was given in the water alone $(P<0 \cdot 001)$.

Liver wet weight differed between groups $(P=0 \cdot 022)$, with group ADW showing the highest weight, while the percentages of water and fat in the liver were similar between the groups (Table 2). Moreover, epididymal adipose tissue weight of the group ADW was significantly higher than that of the other groups $(P=0.007)$ and positively correlated with the amount of ingested aspartame $(r=0.559, P=0.002)$. The weights of the kidneys and hearts of all rats were found to be similar, with no significant differences observed between the four groups (online Supplementary Table 2).

At the blood metabolites level, fasting plasma glucose was significantly higher among the aspartame groups $(P=0.003)$ and this seems to relate to the amount of ingested aspartame $(r=0.585, P=0.001)$ (Table 2). Fasting plasma insulin levels failed to reach significance between the groups $(P=0 \cdot 125)$, whereas HOMA-IR was significantly different between groups $(P=0.04)$ and strongly related to the amount of ingested aspartame $(r=0.518, P=0.006)$, and thus insulin sensitivity was inversely related to aspartame intake. Fasting plasma TAG levels were also significantly different between the groups $(P=0.039)$, in which $\mathrm{AD}$ and $\mathrm{ADW}$ had the highest levels. As for the remaining parameters, no significant differences were detected between all four groups for total cholesterol, HDL, albumin, plasma urea nitrogen and creatinine levels (online Supplementary Table 2).

\section{Expt 2: Effect of sucralose ingestion from water and/or diet on body weight measures and blood metabolites}

Body weight gain showed statistical significance according to time $(P=0 \cdot 001)$ and groups $(P<0 \cdot 001)$, mainly due to a higher weight gain in group SDW as compared with sD (Fig. 2(a)). Similarly, fat mass gain was significant according to time $(P=0.001)$ and groups $(P<0.001)$. Fat gain of the SDW group was significantly higher than that of the SW group (Fig. 2(b)), whereas lean body mass gain was found similar between the varied groups $(P=0.474)$ (Fig. 2(c)). However, no significant correlation was detected between the amount of ingested sucralose and body weight gain, fat mass gain, or lean body mass gain.

Moreover, all measures of energy balance were similar between the groups, with energy intake, expenditure and efficiency failed to reach statistical significance (Table 3). Also, no difference in total fluid intake was observed between the groups (Table 3). Liver weight and composition, as well as epididymal fat weight, were all similar between the groups (Table 4). No statistical significance was detected in any of the blood parameters (Table 4 and online Supplementary Table 3). However, fasting plasma glucose of the sucralose groups was slightly higher than that of the control (Table 4), while insulin and HOMA-IR were similar between the groups. Total and HDL-cholesterol both showed a pattern whereby higher levels were found in the sD group as compared with either the SW or SDW groups, but with no statistical significance detected (online Supplementary Table 3).

\section{Discussion}

NCS have been introduced to diets with the intention of reducing caloric intake and normalising blood glucose levels without compromising diet palatability. They have been extensively used as a substitute for sugar, providing the sweet taste without any energy load, and resulting in short-term reductions in body weight and fat ${ }^{(8,9)}$. However, their use coincides with the increase in the obesity and diabetes epidemics, and long-term studies associated them with weight gain and designated them as a factor fuelling obesity ${ }^{(11,12,16-19)}$. Indeed, prolonged consumption of foods or fluids containing acesulfame-K or saccharin, in comparison with glucose, has led to an increase in food intake, body weight and body fat accumulation in rats ${ }^{(20,21)}$. Our previous work further supported this notion, as both body weight and fat gains were found to be higher in rats consuming acesulfame-K as compared with sucrose-sweetened water ${ }^{(22)}$. Meanwhile, the ingestion of dietary carbohydrates in varied forms (solid food or liquid beverages) was reported to influence metabolic processing and body composition differently ${ }^{(13)}$. Accordingly, we designed an experiment to investigate the 
(a)

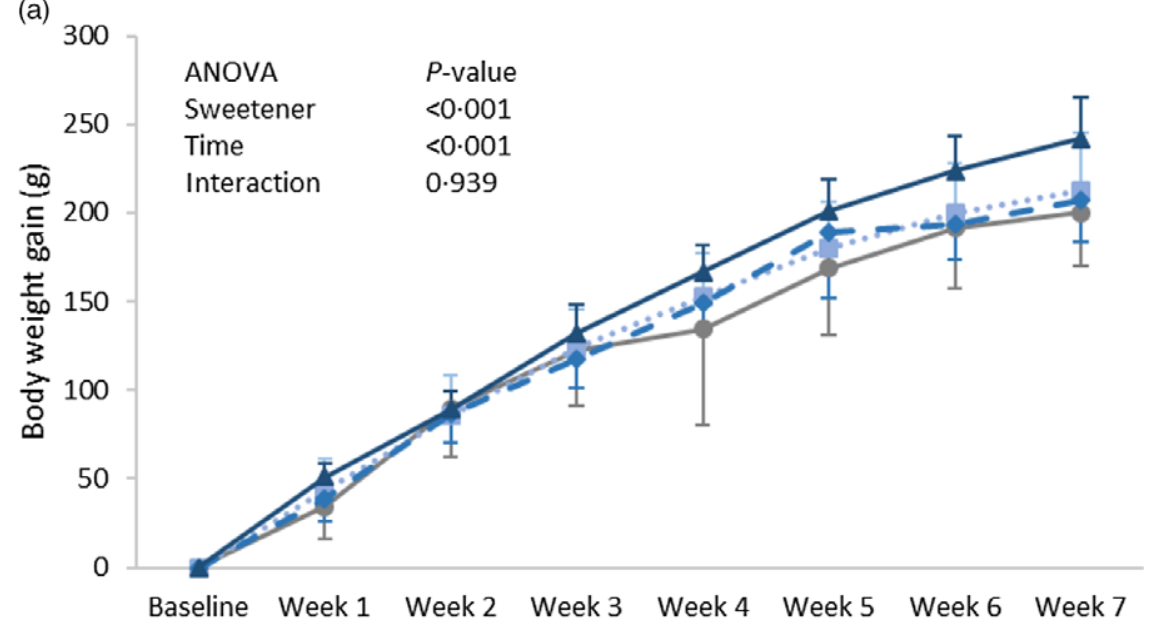

(b)

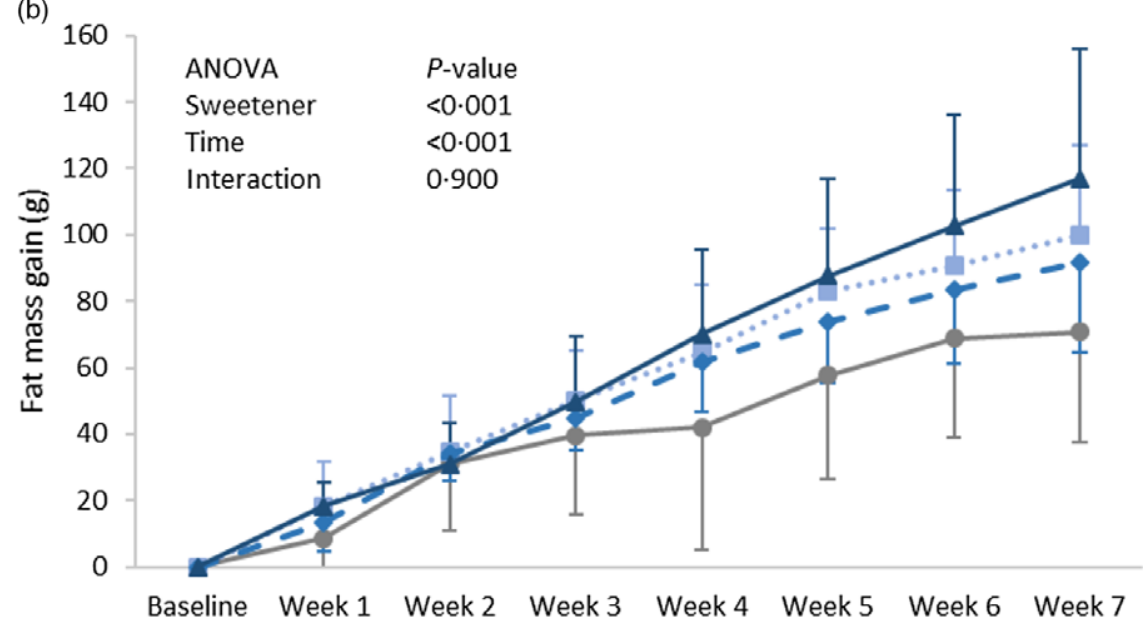

(c)

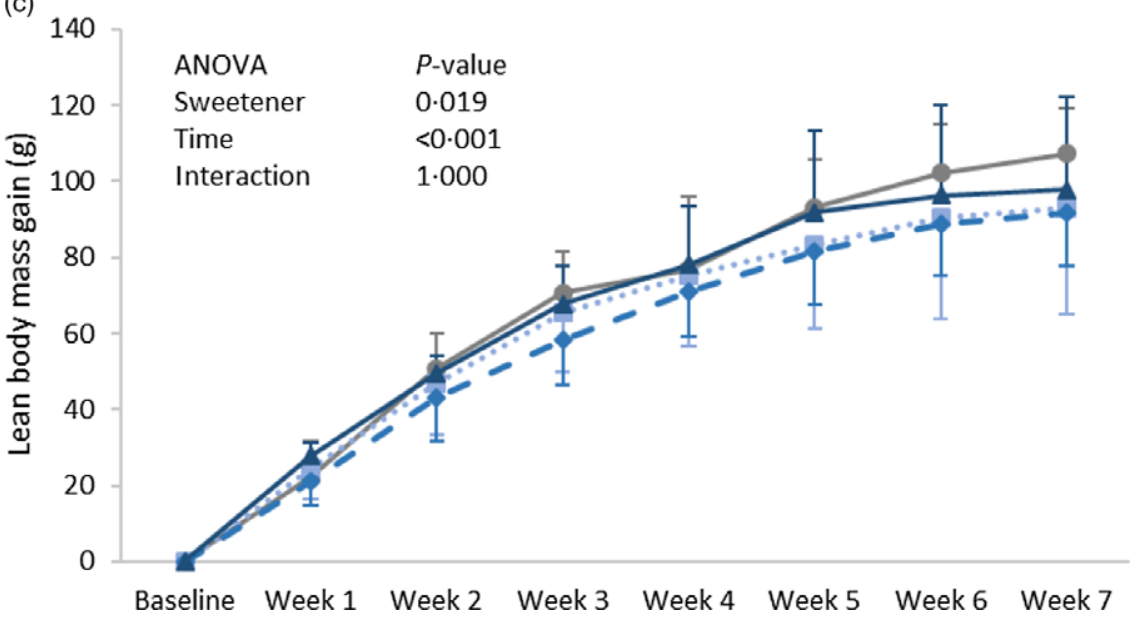

Fig. 1. Expt 1 - weekly body weight (a), lean body mass (b) and body fat (c) of the four groups of rats in Expt 1 over the 8-week experimental period. Group $C$ - control: starch-based diet and plain water; Group AD: aspartame-sweetened diet and plain water; Group AW: starch-based diet and aspartame-sweetened water; Group ADW: aspartame-sweetened diet and water. Data are expressed as the mean \pm SD of all values. A two-way ANOVA was performed with time and group as factors. Significance was set at $P$-value $<0.05 . \rightarrow, \mathrm{C} ; \ldots \ldots, \mathrm{AD} ; \multimap-, \mathrm{AW} ; \multimap, \mathrm{ADW}$.

impact of the ingestion of NCS (aspartame and sucralose) in varied forms on body weight measures. In the current study, the amount of sweeteners used were set to equal the sweetness level of a $10 \%$ glucose solution, a common level used in beverages.
Aspartame ingestion for 7 weeks was able to increase body weight gain, in which gain was positively associated with the amount of ingested aspartame rather than the form of aspartame (water or diet) ingestion. The increase in weight gain was highly 
Table 1. Expt 1 - effect of aspartame ingestion from water and/or diet on measures of energy balance

\begin{tabular}{|c|c|c|c|c|c|c|c|c|c|}
\hline & \multicolumn{2}{|c|}{ C } & \multicolumn{2}{|c|}{$A D$} & \multicolumn{2}{|c|}{ AW } & \multicolumn{2}{|c|}{ ADW } & \multirow[t]{2}{*}{$P$} \\
\hline & Mean & SD & Mean & SD & Mean & SD & Mean & $\mathrm{SD}$ & \\
\hline Energy intake $(\mathrm{kJ} / \mathrm{d})$ & 453.34 & 33.97 & 472.42 & 16.95 & 484.55 & 51.30 & 476.39 & 35.98 & 0.496 \\
\hline Fluid intake (g/d) & 44.08 & 20.96 & 32.89 & 4.24 & 39.61 & $10 \cdot 87$ & 38.30 & $5 \cdot 13$ & 0.409 \\
\hline Plain water intake $(\mathrm{g} / \mathrm{d})$ & 44.08 & $20.96^{\mathrm{a}}$ & 32.89 & $4 \cdot 24^{\mathrm{a}}$ & $20 \cdot 10$ & $4 \cdot 27^{\mathrm{b}}$ & 19.58 & $2 \cdot 20^{\mathrm{b}}$ & 0.001 \\
\hline Sweet water intake $(\mathrm{g} / \mathrm{d})$ & 0.00 & $0.00^{\mathrm{a}}$ & 0.00 & $0.00^{\mathrm{a}}$ & 19.51 & $7 \cdot 19^{\mathrm{b}}$ & $18 \cdot 72$ & $3.70^{b}$ & 0.001 \\
\hline Sweetener intake $(\mathrm{mg} / \mathrm{d})$ & 0.00 & $0.00^{\mathrm{a}}$ & 14.86 & $0.53^{b}$ & 9.76 & $3 \cdot 60^{c}$ & $24 \cdot 34$ & $2.08^{\mathrm{d}}$ & 0.001 \\
\hline Energy expenditure $(\mathrm{kJ} / \mathrm{d})$ & 387.48 & 38.74 & 384.13 & 22.51 & 403.09 & 42.05 & $374 \cdot 18$ & 50.75 & 0.601 \\
\hline Energy stored (kJ/100kJ) & 14.51 & 5.57 & 17.94 & 4.00 & $16 \cdot 73$ & 3.58 & 21.62 & 6.64 & 0.107 \\
\hline
\end{tabular}

Group C - control: starch-based diet and plain water; Group AD: aspartame-sweetened diet and plain water; Group AW: starch-based diet and aspartame-sweetened water; Group ADW: aspartame-sweetened diet and water.

Data are expressed as the mean \pm SD of all values. A one-way ANOVA was performed, data with the same subscript are not significantly different according to Fisher's pairwise comparison.

Significance was set at a $P$-value $<0.05$.

Table 2. Expt 1 - effect of aspartame ingestion from water and/or diet on weight of organs and plasma metabolites

\begin{tabular}{|c|c|c|c|c|c|c|c|c|c|}
\hline & \multicolumn{2}{|c|}{ C } & \multicolumn{2}{|c|}{$A D$} & \multicolumn{2}{|c|}{ AW } & \multicolumn{2}{|c|}{ ADW } & \multirow[b]{2}{*}{$P$} \\
\hline & Mean & SD & Mean & SD & Mean & SD & Mean & SD & \\
\hline Liver wet weight (g) & $12 \cdot 48$ & $0.69^{\mathrm{a}}$ & $14 \cdot 37$ & $2 \cdot 15^{\mathrm{ab}}$ & $12 \cdot 61$ & $1.91^{\mathrm{a}}$ & 15.53 & $2 \cdot 38^{\mathrm{b}}$ & 0.022 \\
\hline Liver weight $\%$ of BW (g/100g BW) & 2.54 & 0.13 & 2.73 & 0.26 & $2 \cdot 50$ & 0.24 & $2 \cdot 78$ & 0.25 & 0.085 \\
\hline Liver water (\%) & 67.52 & $2 \cdot 15$ & 67.55 & $2 \cdot 36$ & 66.06 & 3.87 & $67 \cdot 71$ & 1.01 & 0.514 \\
\hline Liver fat in wet (\%) & 5.84 & 1.86 & 5.48 & 1.36 & 6.31 & $1 \cdot 16$ & $7 \cdot 21$ & 0.86 & 0.118 \\
\hline Epididymal AT weight (g) & 8.78 & $2 \cdot 46^{\mathrm{a}}$ & 11.52 & $2 \cdot 12^{\mathrm{a}}$ & 11.08 & $2 \cdot 06^{\mathrm{a}}$ & 14.55 & $3.62^{b}$ & 0.007 \\
\hline Fasting plasma glucose (mg/dl) & 166.67 & $40 \cdot 76^{a}$ & $244 \cdot 14$ & $48 \cdot 42^{\mathrm{bc}}$ & 218.00 & $47 \cdot 30^{\mathrm{ab}}$ & $268 \cdot 14$ & $41.50^{c}$ & 0.003 \\
\hline Fasting plasma insulin ( $\mathrm{ng} / \mathrm{ml})$ & 0.55 & 0.74 & 1.02 & 0.65 & 0.58 & 0.72 & 1.39 & 0.73 & 0.125 \\
\hline HOMA-IR & 7.41 & $11 \cdot 10^{\mathrm{b}}$ & 17.73 & $10 \cdot 26^{\mathrm{ab}}$ & 9.72 & $13 \cdot 50^{\mathrm{b}}$ & $25 \cdot 61$ & $12 \cdot 23^{a}$ & 0.040 \\
\hline Fasting plasma TAG (mg/dl) & $46 \cdot 17$ & $21.68^{\mathrm{a}}$ & $65 \cdot 14$ & $28.79^{\mathrm{ab}}$ & 43.14 & $8.43^{\mathrm{a}}$ & 74.57 & $22 \cdot 87^{\mathrm{b}}$ & 0.039 \\
\hline
\end{tabular}

BW, body weight; AT, adipose tissue; HOMA-IR, Homeostatic Model Assessment for Insulin Resistance.

Group C - control: starch-based diet and plain water; Group AD: aspartame-sweetened diet and plain water; Group AW: starch-based diet and aspartame-sweetened water; Group ADW: aspartame-sweetened diet and water.

Data are expressed as the mean \pm SD of all values. A one-way ANOVA was performed, data with the same subscript are not significantly different according to Fisher's pairwise comparison.

Significance was set at a $P$-value $<0.05$.

attributed to total body fat gain. This was also apparent in the varied organs, where group ADW had high liver fat (\%) and epididymal fat pad weight. The similarity in energy intake and energy expenditure between the groups implies that such an increase was related to improved energy efficiency, which was also found to be positively associated with the amount of ingested aspartame. These observations are in line with other studies in which weight gain following long-term consumption of aspartame-sweetened yogurt was not associated with an increase in caloric intake $\mathrm{e}^{(16,23)}$. Thus, it can be concluded that weight gain was highly attributed to an enhancement in energy efficiency.

On the other hand, the impact of sucralose ingestion for 7 weeks was similar to that of aspartame. Body weight measures (weight gain, fat mass gain and lean body mass gain) were increased, though to a lesser magnitude. This may have been behind the failure to show any significant correlation between these parameters and sucralose ingestion. Liver and epididymal fat weight measures also failed to reach any significant difference between groups. Similar to aspartame, sucralose ingestion was not found to affect energy intake and expenditure.

Looking at the figures from both experiments, it is worth noting that the changes in weight and fat gain were manifested from week 3 and this implies that the impact of aspartame and sucralose is not acute and may be related to an up-regulation of parameters involved in lipogenesis and/or fat storage. In fact, the consumption of aspartame-sweetened beverages was linked to extensive liver damage and possible stimulation of hepatic lipogenesis leading to fat deposition in adipose tissues ${ }^{(24)}$. Our findings are in accordance with the numerous studies associating the long-term consumption of NCS with weight gain, further confirming the poor impact of NCS on body composition and metabolism $^{(11,12,18-21)}$. Indeed, NCS were proposed to disrupt the caloric signal associated with sweetness, and thus its failure to provide energy content may consequently enhance energy deposition through the triggering of the starvation mode, which is known to reduce energy expenditure and increase in energy efficiency ${ }^{(12)}$. NCS are actually recognised by the sweet taste receptors that are expressed throughout the body; they however do not seem to be associated with potent satiety signals ${ }^{(25)}$. With animals using sweet taste to predict the caloric contents of food, eating sweet non-caloric substances - that brings the sweet taste without the energy content - will create a positive energy balance through increased food intake and/or diminished energy expenditure ${ }^{(12)}$. Moreover, aspartame and sucralose were reported to have different sites of action on the sweet taste 


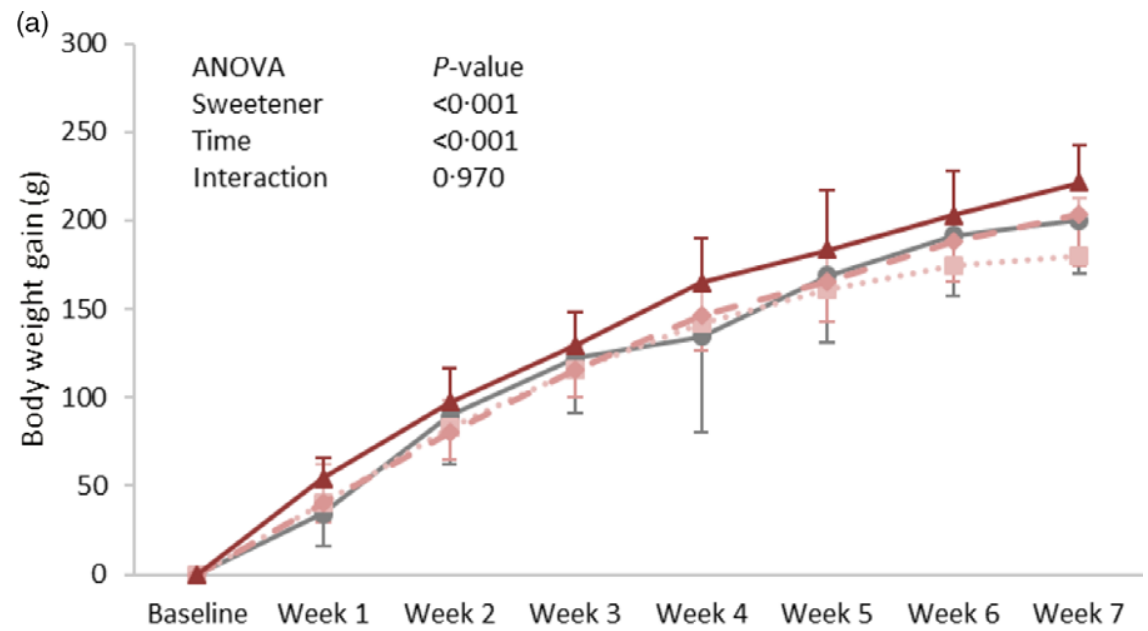

(b)
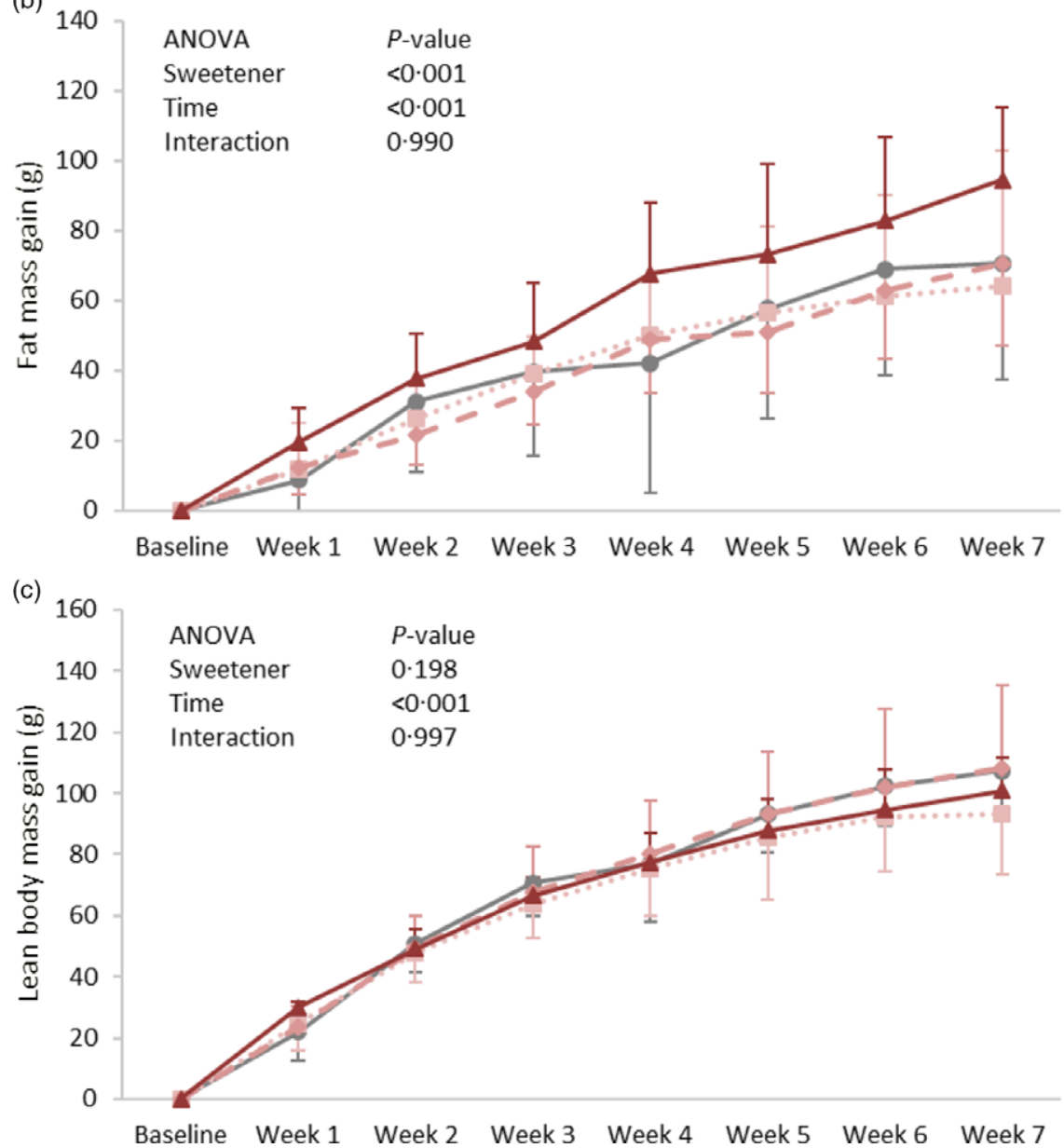

Fig. 2. Expt 2 - weekly body weight (a), lean body mass (b) and body fat (c) of the four groups of rats in Expt 1 over the 8-week experimental period. Group C - control: starch-based diet and plain water; Group sD: sucralose-sweetened diet and plain water; Group SW: starch-based diet and sucralose-sweetened water; Group SDW: sucralose-sweetened diet and water. Data are expressed as the mean and standard deviation of all values. A two-way ANOVA was performed with time and group as factors. Significance was set at $P$-value $<0.05 . \multimap, \mathrm{C} ; \ldots-., \mathrm{SD} ; \multimap, \mathrm{SW} ; \multimap$, SDW.

receptors. Indeed, sucralose, like acesulfame $\mathrm{K}$ and sucrose, bind to both T1R2 and T1R3 sites of the sweet taste receptors, while aspartame only binds to T1R2 $2^{(25)}$. The ability of both aspartame and sucralose to affect body weight and fat raises questions about the involvement of sweet taste receptors in the process of weight gain. In support, the artificial sweeteners saccharin and acesulfame-K were reported to stimulate adipogenesis and suppress lipolysis in a mechanism that is independent of sweet taste receptors $^{(26)}$. Furthermore, the inability of rodents to detect the sweet taste of aspartame, in contrast to that sucralose ${ }^{(25)}$, as well 
Table 3. Expt 2 - effect of sucralose ingestion from water and/or diet on measures of energy balance

\begin{tabular}{|c|c|c|c|c|c|c|c|c|c|}
\hline & \multicolumn{2}{|c|}{ C } & \multicolumn{2}{|c|}{ SD } & \multicolumn{2}{|c|}{ SW } & \multicolumn{2}{|c|}{ SDW } & \multirow[b]{2}{*}{$P$} \\
\hline & Mean & SD & Mean & SD & Mean & SD & Mean & SD & \\
\hline Energy intake $(\mathrm{kJ} / \mathrm{d})$ & $453 \cdot 3$ & 34.0 & 439.9 & 27.5 & $437 \cdot 2$ & $32 \cdot 1$ & 474.0 & $25 \cdot 2$ & 0.118 \\
\hline Fluid intake $(\mathrm{g} / \mathrm{d})$ & 44.08 & 20.96 & 36.57 & 6.85 & 34.10 & 9.71 & 38.23 & $12 \cdot 38$ & 0.599 \\
\hline Plain water intake $(\mathrm{g} / \mathrm{d})$ & 44.08 & $20 \cdot 96^{\mathrm{a}}$ & 36.57 & $6 \cdot 85^{a}$ & $18 \cdot 30$ & $4.37^{\mathrm{b}}$ & 19.88 & $5 \cdot 82^{b}$ & 0.001 \\
\hline Sweet water intake $(\mathrm{g} / \mathrm{d})$ & 0.00 & $0.00^{\mathrm{a}}$ & 0.00 & $0.00^{\mathrm{a}}$ & $15 \cdot 79$ & $6 \cdot 83^{b}$ & 18.35 & $8 \cdot 11^{\mathrm{b}}$ & 0.001 \\
\hline Sweetener intake $(\mathrm{mg} / \mathrm{d})$ & 0.00 & $0.00^{\mathrm{a}}$ & 4.43 & $0 \cdot 0 \cdot 23^{b}$ & $2 \cdot 53$ & $1.09^{c}$ & $7 \cdot 71$ & $1 \cdot 10^{d}$ & 0.001 \\
\hline Energy expenditure $(\mathrm{kJ} / \mathrm{d})$ & 387.5 & 38.7 & 380.6 & $46 \cdot 4$ & 371.3 & $34 \cdot 2$ & 389.6 & $17 \cdot 0$ & 0.769 \\
\hline Energy stored (kJ/100kJ) & 14.51 & 5.57 & $13 \cdot 62$ & 7.04 & 15.09 & 4.05 & 17.73 & $2 \cdot 91$ & 0.493 \\
\hline
\end{tabular}

Group C - control: starch-based diet and plain water; Group SD: sucralose-sweetened diet and plain water; Group SW: starch-based diet and sucralose-sweetened water; Group SDW: sucralose-sweetened diet and water.

Data are expressed as the mean \pm SD of all values. A one-way ANOVA was performed, data with the same subscript are not significantly different according to Fisher's pairwise comparison.

Significance was set at a $P$-value $<0.05$.

Table 4. Expt 2 - effect of sucralose ingestion from water and/or diet on weight of organs and plasma metabolites

\begin{tabular}{|c|c|c|c|c|c|c|c|c|c|}
\hline & \multicolumn{2}{|c|}{ C } & \multicolumn{2}{|c|}{ SD } & \multicolumn{2}{|c|}{ SW } & \multicolumn{2}{|c|}{ SDW } & \multirow[b]{2}{*}{$P$} \\
\hline & Mean & SD & Mean & SD & Mean & SD & Mean & SD & \\
\hline Liver wet weight (g) & 12.48 & 0.69 & 13.49 & $2 \cdot 27$ & $13 \cdot 11$ & $2 \cdot 19$ & $15 \cdot 19$ & 3.42 & 0.227 \\
\hline Liver weight $\%$ of $B W(g / 100 \mathrm{~g} \mathrm{BW})$ & 2.54 & 0.13 & 2.73 & 0.30 & 2.65 & 0.28 & 2.82 & 0.45 & 0.440 \\
\hline Liver water (\%) & 67.52 & $2 \cdot 15$ & 67.61 & 2.78 & 70.85 & 5.34 & $71 \cdot 26$ & $6 \cdot 29$ & 0.320 \\
\hline Liver fat in wet (\%) & 5.84 & 1.86 & $6 \cdot 18$ & 2.49 & 5.88 & 3.57 & 5.71 & 1.76 & 0.990 \\
\hline Epididymal AT weight (g) & 8.78 & 2.46 & $10 \cdot 00$ & 3.52 & 8.87 & $2 \cdot 10$ & $12 \cdot 08$ & 3.02 & 0.137 \\
\hline Fasting plasma glucose (mg/dl) & $166 \cdot 7$ & 40.8 & $204 \cdot 0$ & 32.0 & 207.6 & 39.3 & 258.0 & $93 \cdot 3$ & 0.068 \\
\hline Fasting plasma insulin $(\mathrm{ng} / \mathrm{mL})$ & 0.55 & 0.74 & 1.03 & 0.88 & 0.67 & 1.09 & 0.99 & 1.01 & 0.758 \\
\hline HOMA-IR & 7.41 & $11 \cdot 10$ & $17 \cdot 23$ & 14.96 & 11.32 & 20.38 & 17.56 & 16.85 & 0.646 \\
\hline Fasting plasma TAG (mg/dl) & 46.17 & 21.68 & 40.00 & $17 \cdot 11$ & 35.29 & 9.03 & 51.57 & 20.78 & 0.367 \\
\hline
\end{tabular}

BW, body weight; AT, adipose tissue, HOMA-IR, Homeostatic Model Assessment for Insulin Resistance.

Group C - control: starch-based diet and plain water; Group sD: sucralose-sweetened diet and plain water; Group SW: starch-based diet and sucralose-sweetened water; Group SDW: sucralose-sweetened diet and water.

Data are expressed as the mean \pm SD of all values. A one-way ANOVA was performed, data with the same subscript are not significantly different according to Fisher's pairwise comparison.

Significance was set at a $P$-value $<0.05$.

as the failure of both aspartame and sucralose to increase caloric intake or decrease energy expenditure, do not support the concept of sweet-caloric detection ${ }^{(12)}$. It is worth noting that in both experiments the ingestion of aspartame or sucralose (whether from water and/or diet) was not found to affect the proportion of fluid intake from plain to sweetened water, in contrast to that reported previously, in which caloric (sucrose) and non-caloric (acesulfame K)-sweetened water intake was highly favoured over plain water ${ }^{(22)}$. In the current study, the mechanism by which NCS affect body weight seems to relate to an improvement in energy efficiency, though the mechanism behind such improvement remains to be elucidated.

Insulin sensitivity $(r=0.518, P=0.006)$ was negatively and both fasting plasma glucose $(r=0.585, P=0.001)$ and insulin ( $r=0.442, P=0.021$ ) were positively correlated with aspartame ingestion. These changes may have contributed to the increase in plasma TAG $(r=0.437 P=0.023)$ and epididymal fat pad weight $(r=0.559, \mathrm{P}=0.002)$. However, the modest increase in plasma glucose of the sucralose groups did not reach statistical significance. As previously mentioned, the consumption of NCS was intended as a substitute to sugar that does not affect glycaemia ${ }^{(25)}$, but recent data reported conflicting evidence and associated NCS consumption with increased blood glucose levels and risk of type 2 diabetes $^{(17,27)}$. While the mechanisms responsible for the impact of NCS on body metabolism remain unclear, many theories have been laid to explain the adverse effect on glucose homoeostasis. On the one hand, aspartame consumption in water by mice was reported to inhibit endogenous intestinal alkaline phosphatase activity, which is known to prevent the metabolic syndrome, thus leading to increment in weight gain and plasma glucose, impaired glucose tolerance, and increased TNF- $\alpha$ levels ${ }^{(28)}$. Additionally, recent data are focusing on the possible involvement of circulating metabolites and alternations to the gut microbiota as driving factors behind the effect of NCS on glucose metabolism ${ }^{(28,29)}$. Chronic high doses of aspartame in rats induced an increase in fasting glycaemia and an impairment in insulin-stimulated glucose disposal. These were paralleled with alterations in the gut microbiota and an increase in SCFA propionate, a highly gluconeogenic substrate associated with inflammation and insulin resistance ${ }^{(29)}$. The observed increase in liver fat content in the ADW group may also partially contribute to aspartames' reported suspicion of inflicting damages on many organs, notably the liver, through its role in provoking oxidative stress and increasing free radical production and pro-inflammatory cytokines $^{(30)}$. 
In conclusion, 7-week aspartame ingestion was found to increase body weight gain mainly due to fat accumulation, more specifically from week three and onwards. These effects were not accompanied by changes in energy intake or expenditure and thus relate to an enhancement in energy efficacy. Additionally, insulin sensitivity was reduced due to an increase in both glucose and insulin levels, while liver and epididymal fat weights were increased. These changes were dose-dependent, and thus with the differences observed in the intake of sweeteners (both aspartame and sucralose) between groups, the impact of the form of ingestion (diet and/or water) remains unclear. Sucralose was found to have a similar effect to that of aspartame, though to a lower extent. Our findings highlight the adverse effects of NCS aspartame and sucralose on body composition and metabolism.

\section{Acknowledgements}

This research received no specific grant from any funding agency, commercial or not-for-profit sectors.

O. A. O. conceived and designed the experiment; M. E. R., R. E. and F. E. performed the experiments and conducted the laboratory analysis; M. E. R., R. E. and O. A. O. analysed the data; M. E. R., R. E. and O. O. were involved in writing the paper, and all authors had final approval of the submitted and published versions of the manuscript.

There are no conflicts of interest.

\section{Supplementary material}

For supplementary material/s referred to in this article, please visit https://doi.org/10.1017/S0007114521003238

\section{References}

1. Striegel-Moore RH, Thompson D, Affenito SG, et al. (2006) Correlates of beverage intake in adolescent girls: the National Heart, Lung, and Blood Institute Growth and Health Study. J Pediatr 148, 183-187.

2. Hu FB \& Malik VS (2010) Sugar-sweetened beverages and risk of obesity and type 2 diabetes: epidemiologic evidence. Physiol Behav 100, 47-54.

3. Hu FB (2013) Resolved: there is sufficient scientific evidence that decreasing sugar-sweetened beverage consumption will reduce the prevalence of obesity and obesity-related diseases. Obes Rev 14, 606-619.

4. James J \& Kerr D (2005) Prevention of childhood obesity by reducing soft drinks. Int J Obes 29, S54-S57.

5. Vartanian LR, Schwartz MB \& Brownell KD (2007) Effects of soft drink consumption on nutrition and health: a systematic review and meta-analysis. Am J Public Health 97, 667-675.

6. Brand-Miller JC \& Barclay AW (2017) Declining consumption of added sugars and sugar-sweetened beverages in Australia: a challenge for obesity prevention. Am J Clin Nutr 105, 854-863.

7. Barclay AW \& Brand-Miller J (2011) The Australian paradox: a substantial decline in sugars intake over the same timeframe that overweight and obesity have increased. Nutrients $\mathbf{3}$, 491-504.

8. De la Hunty A, Gibson S \& Ashwell M (2006) A review of the effectiveness of aspartame in helping with weight control. Nutr Bull 31, 115-128.

9. de Ruyter JC, Olthof MR, Seidell JC, et al. (2012) A trial of sugar-free or sugar-sweetened beverages and body weight in children. N Engl J Med 367, 1397-1406.

10. Fagherazzi G, Vilier A, Sartorelli DS, et al. (2013) Consumption of artificially and sugar-sweetened beverages and incident type 2 diabetes in the Etude Epidémiologique auprès des femmes de la Mutuelle Générale de l'Education Nationale-European Prospective Investigation into Cancer and Nutrition cohort. Am J Clin Nutr 97, 517-523.

11. Fowler SP, Williams K, Resendez RG, et al. (2008) Fueling the obesity epidemic? Artificially sweetened beverage use and long-term weight gain. Obesity 16, 1894-1900.

12. Swithers SE (2013) Artificial sweeteners produce the counterintuitive effect of inducing metabolic derangements. Trends Endocrinol Metab 24, 431-441.

13. DiMeglio DP \& Mattes RD (2000) Liquid $v$. solid carbohydrate: effects on food intake and body weight. Int J Obes $\mathbf{2 4}$, 794-800.

14. Ravussin Y, Gutman R, LeDuc CA, et al. (2013) Estimating energy expenditure in mice using an energy balance technique. Int J Obes 37, 399.

15. Matthews DR, Hosker J, Rudenski A, et al. (1985) Homeostasis model assessment: insulin resistance and $\beta$-cell function from fasting plasma glucose and insulin concentrations in man. Diabetologia 28, 412-419.

16. de Matos Feijó F, Ballard CR, Foletto KC, et al. (2013) Saccharin and aspartame, compared with sucrose, induce greater weight gain in adult Wistar rats, at similar total caloric intake levels. Appetite 60, 203-207.

17. Suez J, Korem T, Zeevi D, et al. (2014) Artificial sweeteners induce glucose intolerance by altering the gut microbiota. Nature 514, 181-186.

18. Bissonnette DJ, List S, Knoblich P, et al. (2017) The effect of nonnutritive sweeteners added to a liquid diet on volume and caloric intake and weight gain in rats. Obesity $\mathbf{2 5}$, $1556-1563$.

19. Blundell JE (2019) Low-calorie sweeteners: More complicated than sweetness without calories. Br J Nutr 125, (8): 934-942. doi: $10.1017 /$ S0007114520003359.

20. Swithers SE, Baker CR \& Davidson T (2009) General and persistent effects of high-intensity sweeteners on body weight gain and caloric compensation in rats. Behav Neurosci 123, 772 .

21. Swithers SE, Martin AA \& Davidson TL (2010) High-intensity sweeteners and energy balance. Physiol Behav 100, 55-62.

22. Ragi M, El-Helou N, El-Mallah C, et al. (2020) Effect of temperature and/or sweetness of beverages on body composition in rats. Br J Nutr 1-9. Br J Nutr. 125(8):934-942. doi: 10.1017/ S0007114520003359.

23. Polyák É, Gombos K, Hajnal B, et al. (2010) Effects of artificial sweeteners on body weight, food and drink intake. Acta Physiologica Hungarica 97, 401-407.

24. Lebda MA, Tohamy HG \& El-Sayed YS (2017) Long-term soft drink and aspartame intake induces hepatic damage via dysregulation of adipocytokines and alteration of the lipid profile and antioxidant status. Nutr Res $\mathbf{4 1}, 47-55$.

25. Fernstrom JD, Munger SD, Sclafani A, et al. (2012) Mechanisms for sweetness. J Nutr 142, 1134S-1141S.

26. Simon BR, Parlee SD, Learman BS, et al. (2013) Artificial sweeteners stimulate adipogenesis and suppress lipolysis 
independently of sweet taste receptors. I Biol Chem $\mathbf{2 8 8}$ 32475-32489.

27. Swithers SE, Laboy AF, Clark K, et al. (2012) Experience with the high-intensity sweetener saccharin impairs glucose homeostasis and GLP-1 release in rats. Behav Brain Res $\mathbf{2 3 3}$ $1-14$.

28. Gul SS, Hamilton ARL, Munoz AR, et al. (2017) Inhibition of the gut enzyme intestinal alkaline phosphatase may explain how aspartame promotes glucose intolerance and obesity in mice. Appl Physiol Nutr Metab 42, 77-83.

29. Palmnäs MS, Cowan TE, Bomhof MR, et al. (2014) Low-dose aspartame consumption differentially affects gut microbiotahost metabolic interactions in the diet-induced obese rat. PloS One 9, e109841.

30. Choudhary AK \& Pretorius E (2017) Revisiting the safety of aspartame. Nutr Rev 75, 718-730. 